BULL. AUSTRAL. MATH. SOC.

VOL. $12(1975), 399-405$.

\title{
On a nonlinear elliptic boundary-value problem
}

\section{E.N. Dancer}

We announce a number of results on the existence of solutions of nonlinear elliptic boundary value problems in the case where the dominating linear part is not invertible. Our theorems improve recent results of Landesman and Lazer, and Williams.

We announce results which improve theorems of Williams [11] and Landesman and Lazer [6] for a nonlinear elliptic boundary-value problem. Williams' result is an improvement on the main theorem in the pioneering paper [5] of Landesman and Lazer.

Suppose that $\Omega$ is a bounded domain in $R^{n}, a_{i j}(i, j=1, \ldots, n)$, and $c$ are in $L^{\infty}(\Omega), a_{i j}=a_{j i}(i, j=1, \ldots, n)$, there is a constant $\mu>0$ such that $a_{i j}(x) \zeta^{i} \zeta^{j} \geq \mu|\zeta|^{2}$ for all $x \in \Omega$ and $\zeta \in R^{n}, f \in L^{2}(\Omega)$ and $g: R \rightarrow R$ is continuous. Then we look for solutions of the equation

$$
L u=g(u)-f
$$

where, for $u \in \dot{w}_{2}^{l}(\Omega), \quad L u=-\frac{\partial}{\partial x_{i}}\left(a_{i j} \frac{\partial u}{\partial x_{j}}\right)+c u$. [Here the derivatives are distributional derivatives.]

Let $\lambda_{1}<\lambda_{2}<\ldots$ denote the distinct eigenvalues of the linear problem $L u=\lambda u, N_{i}=\left\{u \in \dot{W}_{2}^{l}(\Omega): L u=\lambda_{i} u\right\}$, and $h_{1}$ the non-negative

Received 28 January 1975. 
eigenfunction corresponding to $\lambda_{1}$.

We say that $k: R \rightarrow R$ satisfies Property $\mathrm{P}$ if $\lim k(x)$ and $x \rightarrow \infty$

lim $k(x)$ both exist. (where the limits may be $\pm \infty$ ) and there exist $x \rightarrow-\infty$

$r, d>0$ such that $|k(s x)| \geq r|k(x)|-d$ if $|x| \geq d$ and $s \geq d$. We

say that Assomption $S$ holds if $\Omega$ has $c^{2}$ boundary and there is a compact subset $T$ of $\Omega$ such that $a_{i j}(i, j=1, \ldots, n)$ are Lipschitz continuous on $\bar{\Omega} T$.

THEOREM 1. Suppose that there is a positive integer $i$ such that $g(x)-\lambda_{i} x$ satisfies Property $P, \quad \lambda_{i} \leq \underset{|x| \rightarrow \infty}{\lim \inf } x^{-1} g(x)$ $\lim _{x \rightarrow \infty} \sup x^{-1} g(x) \leq \lambda_{i+1}, \lim _{x \rightarrow-\infty} \sup x^{-1} g(x) \leq \lambda_{i+1}$ and one of the last two inequalities is a strict inequality. Let $I^{ \pm}=\lim _{x \rightarrow \pm \infty}\left(g(x)-\lambda_{i} x\right)$. In addition, assume that one of the following three conditions holds:

(a) $i>1$, at least one of $I^{+}$and $I^{-}$is infinite, $I^{+} \neq I^{-}$ and $f \in L^{p}(\Omega)$ where $p>\max \left\{2, \frac{3}{2} n\right\}$;

(b) $i=1$, Assumption $S$ holds, $f \in I^{p}(\Omega)$ where $p>\max \{2, n\}$ $\int_{\Omega} f h_{1} d x / \int_{\Omega} h_{1} d x$ lies strictly between $I^{+}$and $I^{-}$;

(c) $I^{+}$and $I^{-}$are both finite, $f \in L^{2}(\Omega)$ and either

(i) $I^{+}>I^{-}$and

$$
I^{+} \int_{\Omega^{+}} h d x+I^{-} \int_{\Omega^{-}} h d x>\int_{\Omega} f h d x
$$

for all $h \in N_{i}$, or

(ii) $I^{-}>I^{+}$and

$$
I^{-} \int_{\Omega^{+}} h d x+I^{+} \int_{\Omega^{-}} h d x>\int_{\Omega} f h d x
$$




$$
\begin{aligned}
& \text { for all } h \in N_{i} \quad\left(\text { where } \Omega^{+}=\{x \in \Omega: h(x)>0\}\right. \text { and } \\
& \left.\Omega^{-}=\{x \in \Omega: h(x)<0\}\right) \text {. }
\end{aligned}
$$

Then equation (1) has a solution.

In (b), we may omit Assumption sprovided that we replace " $\geq d$ " by $"|s| \geq d "$ in the definition of Property P. If $\underset{x \rightarrow \infty}{\lim \inf } x^{-1} g(x)>\lambda_{i}$ or $\underset{x \rightarrow-\infty}{\lim } \inf x^{-1} g(x)>\lambda_{i}$, we need not assume that $I^{+} \neq I^{-}$in $(a)$ and we may

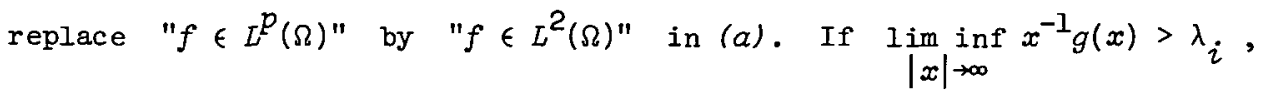
we may replace " $f \in L^{p}(\Omega)$ " by " $f \in L^{2}(\Omega)$ " in (b). In $(c)$, we may replace ">" by " $\geq$ " in condition $\left(^{*}\right)$ and replace $" I^{-}>I^{+}$" by $" I^{-} \geq I^{+} "$ if we assume that $f \in L^{p}$ where $p>\max \left\{2, z_{2} n\right\}$ and $\lim _{x \rightarrow \pm \infty} x\left(g(x)-\lambda i_{i} x-I^{ \pm}\right)<0$. This improves the result in [2]. The last

condition can be weakened under further regularity assumptions on $L, \partial \Omega$, and $f$.

The case where $I^{-}>I^{+}$and the assumptions of (c) are satisfied is essentially the result in [11] while the case where

$$
\lambda_{i}<\lim _{|x| \rightarrow \infty} \inf x^{-1} g(x) \leq \underset{|x| \rightarrow \infty}{\lim \sup } x^{-1} g(x)<\lambda_{i+1}
$$

is the result in [6].

The proof of Theorem 1 depends on two lemmas which are of independent interest. By a scalar product on a Banach space $X$, we mean a symetric bilinear mapping from $X \times X$ to $R$.

LEMMA 1. Suppose that $X$ and $Y$ are Banach spaces, $A: X \rightarrow Y$ is a continuous linear Fredholm operator of index zero with range $R(A)$ and nulZ-space $N(A), Y_{2}$ is a complement of $R(A)$ in $Y, X_{2}$ is a closed complement of $N(A)$ in $X, Q$ is the projection onto $R(A)$ parallel to $Y_{2}, B$ is a linear isomorphism from $N(A)$ to $Y_{2}$, and $($,$) is a$ scalar product on $Y_{2}$. Assume that $K: X \rightarrow Y$ is completely continuous, $\lim \sup \|Q K(x)\| /\|x\|=M<\infty$ and there exist $\varepsilon, r>0$ such that $\|x\| \rightarrow \infty$ 


$$
\left\langle(I-Q) K\left(x_{1}+x_{2}\right), B x_{1}\right\rangle<0
$$

if $x_{1} \in N(A), \quad x_{2} \in X_{2},\left\|x_{1}\right\| \geq r$ and $\left\|x_{2}\right\| \leq \varepsilon\left\|x_{1}\right\|$. There exists an $r_{1}>0$ such that, if $t \neq 0$ and

$$
|t| M \leq \frac{1}{4} \varepsilon \text { inf }\left\{\|A x\| /\|x\|: x \in X_{2}, x \neq\right. \text { 아, }
$$

then the equation $A u=t K(u)$ has a solution $u$ with $\|u\| \leq r_{1}$ and no solutions with $\|u\|>r_{1}$.

The proof of this lemma is an easy modification of the proof of Theorem 2.6.2 in [8]. In the proof, it is established that a mapping similar to that in the proof of Theorem 2.6.2 in [8] has non-zero degree. Theorem 1 can easily be deduced from Lemma $I$ if $\lim \sup _{|x| \rightarrow \infty}\left|x^{-1} g(x)-\lambda_{i}\right|$ is sufficiently small. The only difficulty is to verify condition (**). This is achieved by some simple estimations. In the proof, we use some of the regularity results in [10] for weak solutions of elliptic partial differential equations.

LEMMA 2. Suppose that $\varepsilon>0$ and that the assumptions of Theorem 1 hold. There exists an $r_{2}>0$ such that, if $u$ is a solution of

$$
L u=\lambda_{i} u+t\left(g(u)-\lambda_{i} u-f\right)
$$

where $\varepsilon \leq t \leq 1$, then $\|u\|_{2} \leq r$ (where \|\|$_{2}$ denotes the usual norm on $\left.L^{2}(\Omega)\right)$

The proof of this resembles the proof of Lemma 4.6 in [1]. Outline of the proof in the case where (a) of Theorem I holds and

$\lim _{x \rightarrow \infty} x^{-1} g(x)<\lambda_{i+1}$. Suppose by way of contradiction that there exist solutions $u_{n}$ of (2) (for $t=t_{n}$ ) such that $\varepsilon \leq t_{n} \leq 1$ for all $n$ and $\left\|u_{n}\right\|_{2} \rightarrow \infty$ as $n \rightarrow \infty$. By choosing a subsequence, we may assume that $\left(\left\|u_{n}\right\|_{2}\right)^{-1} u_{n}$ converges strongly in $\dot{W}_{2}^{l}(\Omega) \cap L^{\infty}(\Omega)$ to $v$ and $t_{n} \rightarrow t^{*}$ as $n \rightarrow \infty$, where $v \neq 0, \varepsilon \leq t^{*} \leq 1$, and

$$
L v=\lambda_{i} v+t^{*} k v
$$


Here $k \in L^{\infty}(\Omega), \quad 0 \leq k(x) \leq \lambda_{i+1}-\lambda_{i}$ on $\Omega$ and $k(x)<\lambda_{i+1}-\lambda_{i}$ when $v(x)>0$. (In showing that $v$ satisfies (3), we show that

$\left(\left\|u_{n}\right\|_{2}\right)^{-1}\left(g\left(u_{n}\right)-\lambda_{i} u_{n}\right)$ converges weakly to $k$ in $L^{2}(\Omega)$.) By comparison arguments, it is shown that (3) is impossible unless $v \in N_{i}$. In this case, we obtain a contradiction by noting that

$$
\int_{\Omega}\left(g\left(u_{n}\right)-\lambda_{i} u_{n}\right) v d x=\int_{\Omega} f v d x
$$

and then using the inequalities needed to verify condition (**) of Lemma 1 .

The proof of Theorem 1 can now be completed by constructing a similar mapping to that in the proof of Theorem 2.6.2 in [8] and then using Lemma 1, a remark after Lemma 1 , Lemma 2 and the homotopy invariance of the degree.

THEOREM 2. Suppose that $x^{-1} g(x)+a$ as $x \rightarrow \infty, x^{-1} g(x)+b$ as $x+-\infty$,

$(* * *)$

$$
\left(a-\lambda_{i}\right) \int_{\Omega^{+}} h^{2} d x+\left(b-\lambda_{i}\right) \int_{\Omega^{-}} h^{2} d x>0 \quad(<0)
$$

for every $h \in N_{i}$, and $\left|a-\lambda_{i}\right|+\left|b-\lambda_{i}\right|$ is sufficiently small. Then (1) has a solution for every $f \in L^{2}(\Omega)$.

This follows easily from Lemma 1. This result should be contrasted with Theorem 3.1 in [1]. On the other hand, if $N_{i}=\{\alpha h: \alpha \in R\}$ and condition (***) is replaced by:-

$$
\left(a-\lambda_{i}\right) \int_{\Omega^{+}} h^{2} d x+\left(b-\lambda_{i}\right) \int_{\Omega^{-}} h^{2} d x
$$

and

$$
\left(a-\lambda_{i}\right) \int_{\Omega^{-}} h^{2} d x+\left(b-\lambda_{i}\right) \int_{\Omega^{+}} h^{2} d x
$$

are both non-zero and have opposite signs, it can be shown that $\left\{f \in L^{2}(\Omega):(I)\right.$ has a solution $\}$ is a closed proper subset of $L^{2}(G)$. (If $i=1$, we need only assume that $a<\lambda_{1}<b \leq \lambda_{2}$ or 
$\left.b<\lambda_{1}<a \leq \lambda_{2} \cdot\right)$

THEOREM 3. Let $I^{+}=\lim _{x \rightarrow \infty} \sup \left(g(x)-\lambda_{1} x\right)$ and

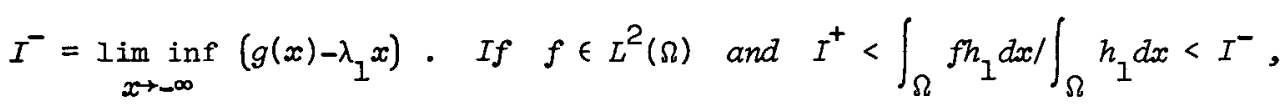

then (1) has a solution.

This result can easily be proved by using the results in [4]. Alternatively, it can be proved by applying Theorem 1 to a truncated equation and then passing to the limit. This theorem improves a result in Schatzman [9].

If the assumptions of one of the theorems holds, $f \in L^{p}(\Omega)$ where $p>\frac{n}{2}$, and $u$ is a solution of (1) (with $g(u) \in L^{1}(\Omega)$ if the assumptions of Theorem 3 hold), it can be shown that $u \in L^{\infty}(\Omega)$. It is easy to prove additional regularity results under stronger assumptions. The solutions in Theorems 1 and 3 are unique under additional assumptions on $g$.

Our methods can be applied to a number of other problems. For example, they could be used to improve the main result in [7] and some of the results in [3] on the existence of periodic solutions of ordinary differential equations.

\section{References}

[1] Antonio Ambrosetti, Giovanni Prodi, "On the inversion of some differentiable mappings with singularities between Banach spaces", Theory of nonlinear operators, 9-28 (Proc. Summer School, September 1971, Babylon, Czechoslovakia. Academia Publishing House of the Czechoslovak Academy of Sciences, Prague; Academic Press, New York and London; 1973).

[2] Svatopluk Fučík, "Further remark on a theorem of E.M. Landesman and A.C. Lazer", Comment. Math. Univ. Carolinae 15 (1974), 259-271.

[3] Svatopluk Fučík, Vladimír Lovicar, "Boundary value and periodic problem for the equation $x^{\prime \prime}(t)+g(x(t))=p(t)$ ", Comment. Math. Univ. Carolinae 15 (1974), 351-355. 
[4] Peter Hess, "On semi-coercive nonlinear problems", J. Math. Mech. 23 $(1974), 645-654$.

[5] E.M. Landesman \& A.C. Lazer, "Nonlinear perturbations of linear elliptic boundary value problems at resonance", J. Math. Mech. $19(1970), 609-623$.

[6] E.M. Landesman and A.C. Lazer, "Linear eigenvalues and a nonlinear boundary value problem", Pacific J. Math. 33 (1970), 311-328.

[7] A.C. Lazer and D.E. Leach, "Bounded perturbations of forced harmonic oscillators at resonance", Ann. Mat. Pura Appl. 82 (1969), 49-68.

[8] L. Nirenberg, Topics in nonlinear functional analysis (Courant Institute of Mathematical Sciences, New York University, New York, 1973-1974).

[9] MHle Michelle Schatzman, "Problèmes aux limites non linéaires semicoercifs", C.R. Acad. Sci. Paris 275 (1972), 1305-1308.

[10] N.S. Trudinger, "Linear elliptic operators with measurable coefficients", Ann. Scuola. Norm. Sup. Pisa 27 (1973), 265-308.

[11] S.A. Williams, "A sharp sufficient condition for solution of a nonlinear elliptic boundary value problem", J. Differential Equations 8 (1970), 580-586.

\footnotetext{
Department of Mathematics, University of New England, Armidale, New South Wales.
} 